\title{
Plasma density shaping for attosecond electron bunch generation
}

\author{
Andrzej Kornaszewski ${ }^{1}$, Roman Spesyvtsev ${ }^{1}$, Mohammed Shahzad ${ }^{1}$, Enrico \\ Brunetti ${ }^{1}$, Przemysław W. Wachulak ${ }^{2}$, Tomasz Fok ${ }^{2}$, Łukasz Wegrzyński ${ }^{2}$, \\ Giorgio Battaglia ${ }^{1}$, Bernard Ersfeld ${ }^{1}$, James S. Feehan ${ }^{1}$, Lucas I. Inigo Gamiz ${ }^{1}$, \\ Karolina Kokurewicz ${ }^{1}$, Wentao $\mathrm{Li}^{1}$, Antoine Maitrallain ${ }^{1}$, Adam Noble ${ }^{1}$, Lewis R. \\ Reid $^{1}$, Matthew P. Tooley ${ }^{1}$, Gregory Vieux ${ }^{1}$, Samuel M. Wiggins ${ }^{1}$, Sam R. \\ Yoffe $^{1}$, Henryk Fiedorowicz ${ }^{2}$, and Dino A. Jaroszynski ${ }^{1}$ \\ ${ }^{1}$ SUPA, Department of Physics, University of Strathclyde, Glasgow G4 0NG, UK \\ ${ }^{2}$ Institute of Optoelectronics, Military University of Technology, 00-908 Warsaw, \\ Poland
}

\begin{abstract}
High energy attosecond electron bunches from the laser-plasma wakefield accelerator (LWFA) are potentially useful sources of ultra-short duration X-rays pulses, which can be used for ultrafast imaging of electron motion in biological and physical systems. Electron injection in the LWFA depends on the plasma density and gradient, and the laser intensity. Recent research has shown that injection of attosecond electron bunches is possible using a short plasma density ramp. For controlled injection it is necessary to keep both the laser intensity and background plasma density constant, but set to just below the threshold for injection. This ensures that injection is only triggered by an imposed density perturbation; the peak density should also not exceed the threshold for injection. A density gradient that only persists over a short range can lead to the injection of femtosecond duration bunches, which are then Lorentz contracted to attoseconds on injection. We consider an example of a $\sin ^{2}$ shaped modulation where the gradient varies until the downward slope exceeds the threshold for injection and then reduces subsequently to prevent any further injection. The persistence above the threshold determines the injected bunch length, which can be varied. We consider several designs of plasma media including density perturbations formed by shaped Laval nozzles and present an experimental and theoretical study of the modulated media suitable for producing attosecond-duration electron bunches.
\end{abstract}

\section{INTRODUCTION}

Laser plasma acceleration is a relatively new concept for accelerating electrons, proposed by Tajima and Dawson in 1979. ${ }^{1}$ It has advantages over conventional accelerators because the fields

Further author information: (Send correspondence to D.A.J. or A.K.)

AK: andrzej.kornaszewski@strath.ac.uk, D.A.J.: d.a.jaroszynski@strath.ac.uk 


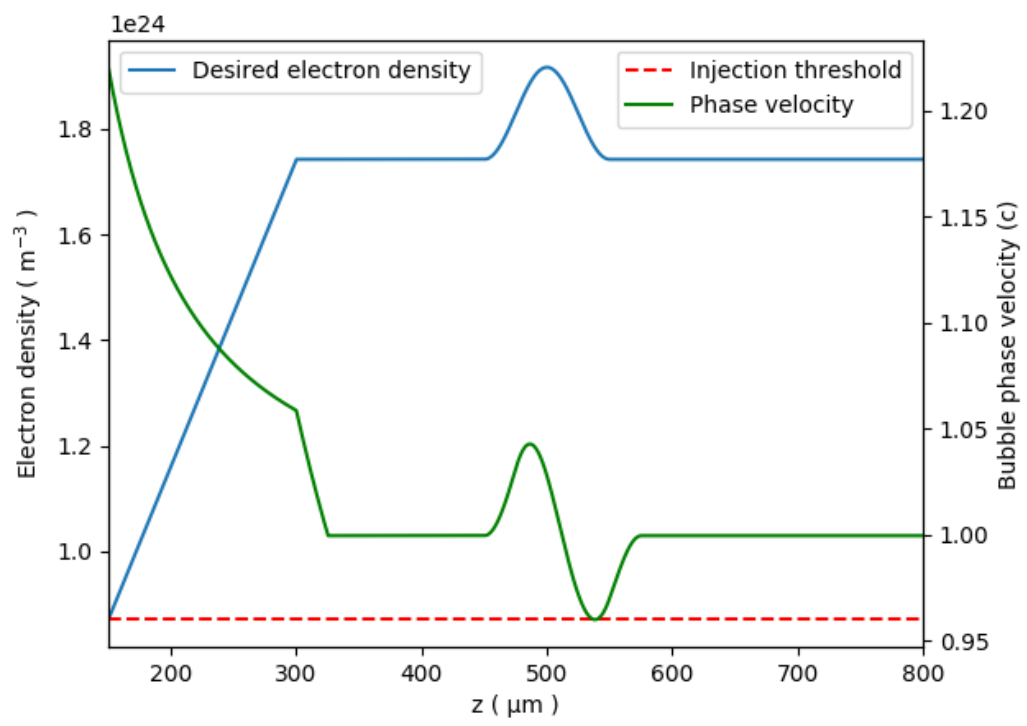

Figure 1. Theoretical injection conditions in a laser-wakefield accelerator. The blue line represents the electron density profile encountered by the laser pulse. The green line represents phase velocity of the back of the bubble. The red dashed line represents the injection threshold. Injection occurs when the phase velocity of the back of the bubble is lower than the injection threshold velocity.

of the acceleration medium, plasma, are not limited by electrical breakdown, and therefore can produce higher acceleration gradients ${ }^{2-6}$. The gradient of a plasma accelerator is only limited by wave breaking, which depends on the plasma density. ${ }^{7}$ The electrostatic fields can be more than three orders of magnitude larger than possible for a classical linear accelerator. This results in a much shorter accelerator for the same energy and therefore decreases the cost of an accelerator. To achieve the largest possible acceleration gradient, the laser intensity should be sufficiently high to reach the so-called "bubble regime", where electrons are fully evacuated from the plasma region trailing the laser pulse, and following injection electrons are accelerated to energies of $\mathrm{MeV}$ to $\mathrm{GeV} .^{2}$

There are several ways for electrons to be injected into the bubble. Tooley et al. ${ }^{8}$ proposed a short persistence down-ramp injection scheme ${ }^{9}$ to briefly inject sub-fs electron bunches. Downramp injection occurs when the velocity of the back of the bubble decreases rapidly, which can be achieved by suddenly reducing the plasma density or when the laser intensity increases. Figure 1 shows how a changing density varies the velocity of the back of the bubble. The condition for injection to occur is when the back of the bubble travels slower than the electron velocity.

This paper investigates methods of producing plasma density profiles suitable for attosecond electron bunch generation and how these can be achieved in the laboratory. We present a design of a gas jet that is capable of attosecond electron bunch injection. Computational fluid dynamics (CFD) simulations are used to determine the feasibility of manufacturing a suitable gas jet. These simulations show that electron density manipulation is possible and the density down-ramp can be controlled. Furthermore, an experimental characterisation of a gas-jet is 
presented. The data from CFD simulations are then used as an input to PIC simulations to demonstrate that the density profiles obtained can generate attosecond electron bunches.

\section{GAS JETS}

The most popular gas targets for laser-wakefield accelerators (LWFAs) are gas jets, gas cells and capillary tubes. Here gas jets are chosen for their ability to generate sharp density gradients, which are required for producing attosecond bunches. Gas jets consist of a nozzle where highpressure gas travels through a shaped or cylindrical hole and exits at supersonic speeds into vacuum. Multiple nozzles can be arranged together to generate complex gas targets. The resulting gas density profile can be tuned by varying the number and spacing of the nozzles, the shape and diameter of the holes, and the distance between the nozzle exits and the laser beam axis.

Several techniques to generate density downramps through density spikes have been investigated. The most successful is a multiple nozzle design. A single nozzle with a cylindrical hole produces a Gaussian density profile. Several closely spaced nozzles, on the other hand, interact with each other and result in a gas structure that resembles the emission from "virtual" gas jets located away from the physical nozzles. These virtual gas jets interact with each other further away, creating density ramps that are suitable for attosecond electron bunch generation. An advantage of using the gas profile generated by the secondary virtual gas jets is the larger distances from the nozzle exit, typically greater than $1.5 \mathrm{~mm}$, which reduces potential damage due to the laser beam or ejected plasma intercepting the nozzle. Furthermore, density spikes produced by secondary virtual gas jets are much sharper than those produced by the primary virtual gas jets.

\section{COMPUTATIONAL FLUID DYNAMICS SIMULATIONS}

Suitable multiple nozzle design has been developed using CFD ANSYS Fluent simulations. To correctly model supersonic gas jets flow, the SST k- $\omega$ turbulence model is used. ${ }^{10}$ This model is best suited to supersonic gases flowing close to solid surfaces. Furthermore, a steady state solution mode is used as it requires substantially reduced CPU times compared with a full transient solver. Simulations are performed in full 3D geometry because multiple nozzles do not have obvious symmetries that allow simplification into a $2 \mathrm{D}$ axisymmetric model. Due to computational limitations a course mesh is used, which is not yet optimised (mesh independent). The simulation volume is minimised to encompass the smallest volume where the boundaries do not affect the region of interest.

Figure 2 shows the density profile of a three nozzle design. The black line represents the axis of a laser beam positioned at a height of $2.6 \mathrm{~mm}$ from the nozzle exit. Figure 3 shows the simulated density profile along this line. To optimise the density distribution for attosecond bunch injection we have investigated its dependence on the gas backing pressure, nozzle separation and nozzle diameter. Gas backing pressure and nozzle diameter mostly determine the overall gas density, the separation distance between nozzles determines the height at which virtual gas jet occurs and the separation distance between jets influences the length of the density plateau on 


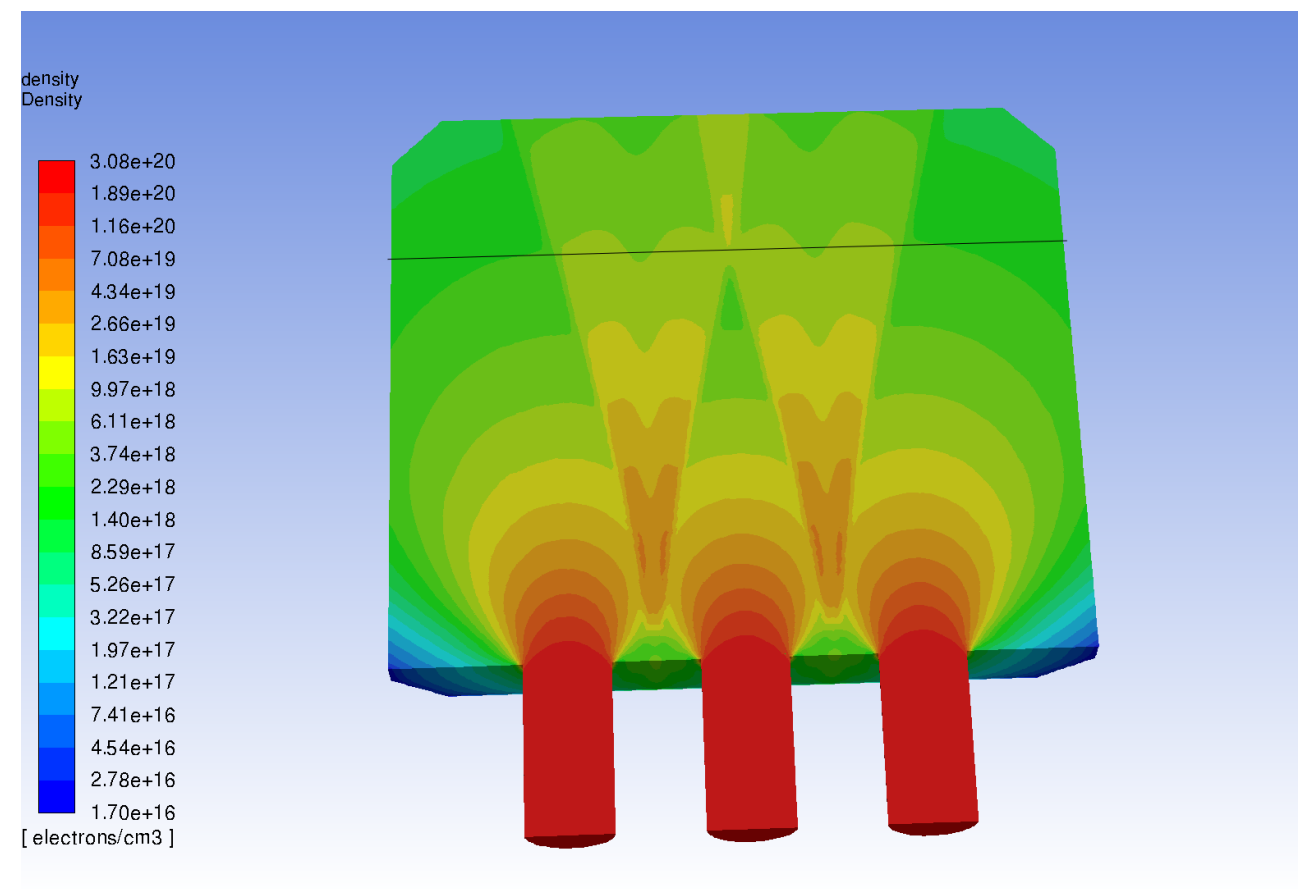

Figure 2. 3-nozzle gas jet simulation. The black line represents a possible laser axis.

each side of the density spike. The design is optimised to maximize the length of the density plateau. The overall density is chosen to achieve electron energies of $100 \mathrm{~s} \mathrm{MeV}$, while remaining just below the self-injection threshold, thus allowing only down-ramp injection.

\section{CHARACTERISATION}

Here we present the experimental characterisation of a 3-nozzles design and compare it with the simulations. The gas density is characterised both interferometry and using shadowgraphy. ${ }^{11}$ However, interferometry was not sensitive enough to detect phase-shifts for low density He gas. We therefore measure a shadowgraph using a quasi-monochromatic source of $13.5 \mathrm{~nm}$ EUV radiation. Figure 4 shows the layout of the shadowgraph experiment. A Nd:YAG laser beam is focused onto a Xenon gas jet of known characteristics ${ }^{12}$ to ionise the gas and produce plasma, which then emits broadband radiation reaching to the EUV spectral region. It is then spectrally filtered using $\mathrm{Zr}$ and $\mathrm{Si}_{3} \mathrm{~N}_{4}$ filters and reflection from a Mo/Si multi-layer mirror to produce a quasi-monochromatic beam of $13.5 \mathrm{~nm}$ radiation, and passed through the gas jet to be characterised. An amount of radiation proportional to the density encountered during propagation is absorbed, thus producing a shadowgram that is recorded on a CCD camera. A simple 2D shadowgram is recorded, although in the future a full 3D tomography will be measured. ${ }^{13}$

A shadowgraph of a helium triple gas jet target with 8 bar backing pressure, has been measured with the gas off and then with gas on. An average of 5 images is recorded, each consisting of 3 shot exposures to minimise data variance. Figure 5 shows the density profile 


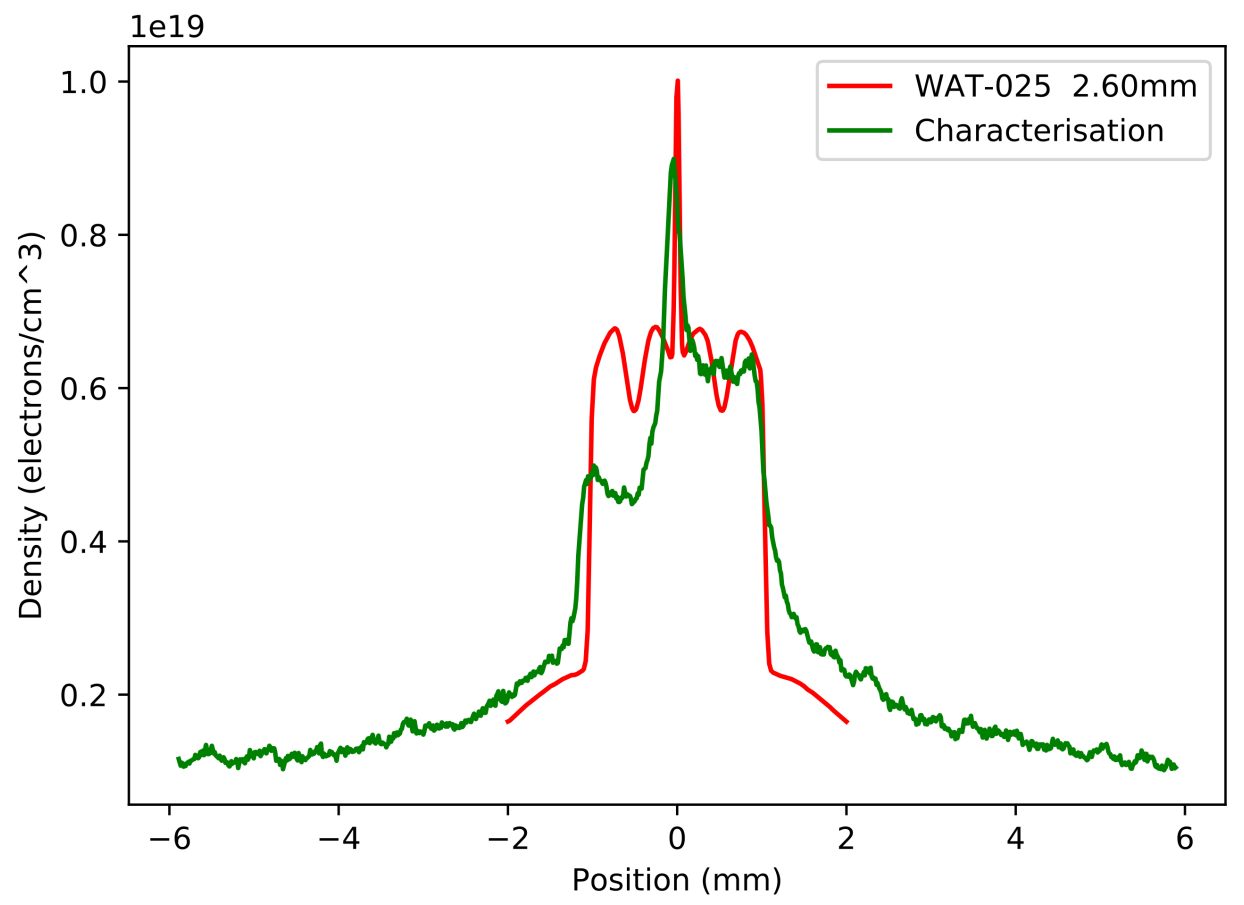

Figure 3. Simulated (red curve) and measured (green curve) density profile, determined $2.6 \mathrm{~mm}$ above the nozzle exit.

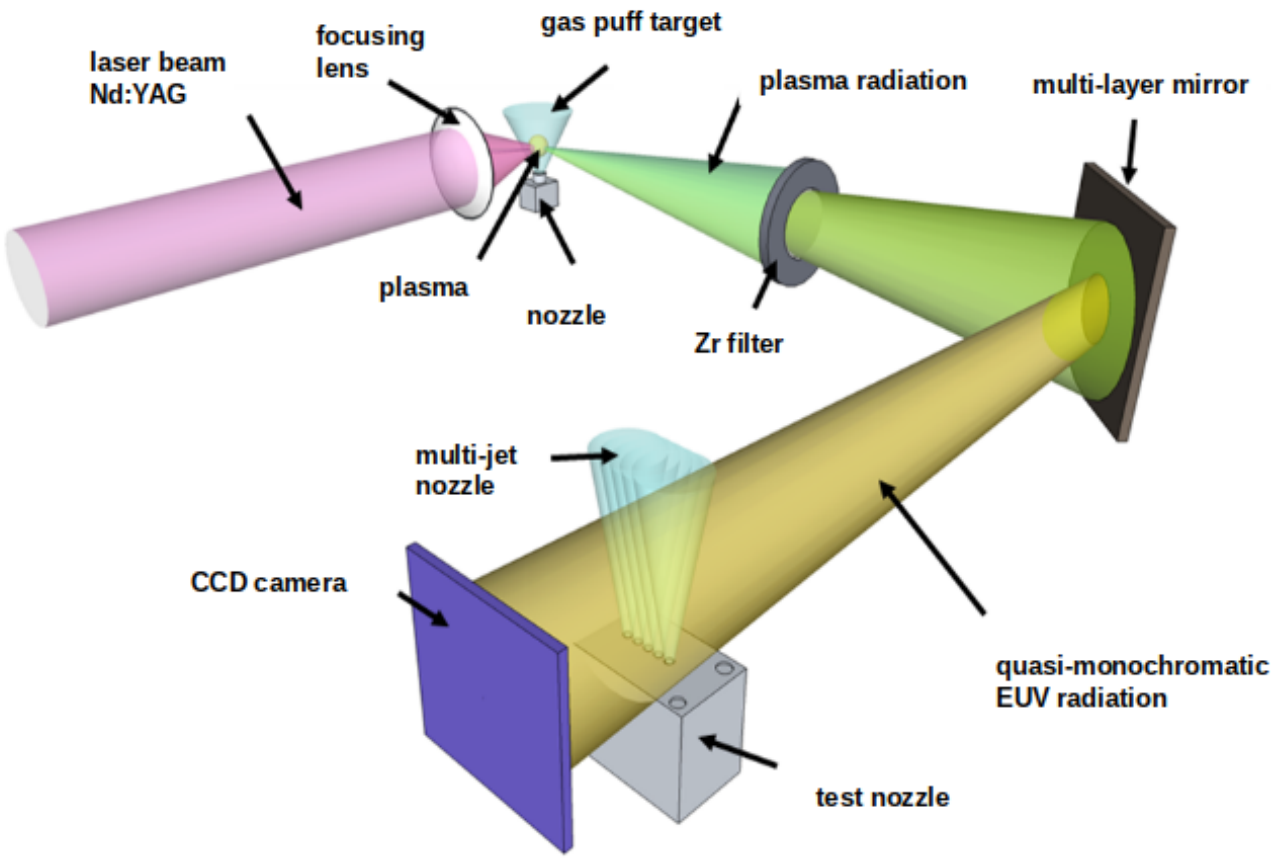

Figure 4. EUV radiography setup for measuring the gas density characterisation. 


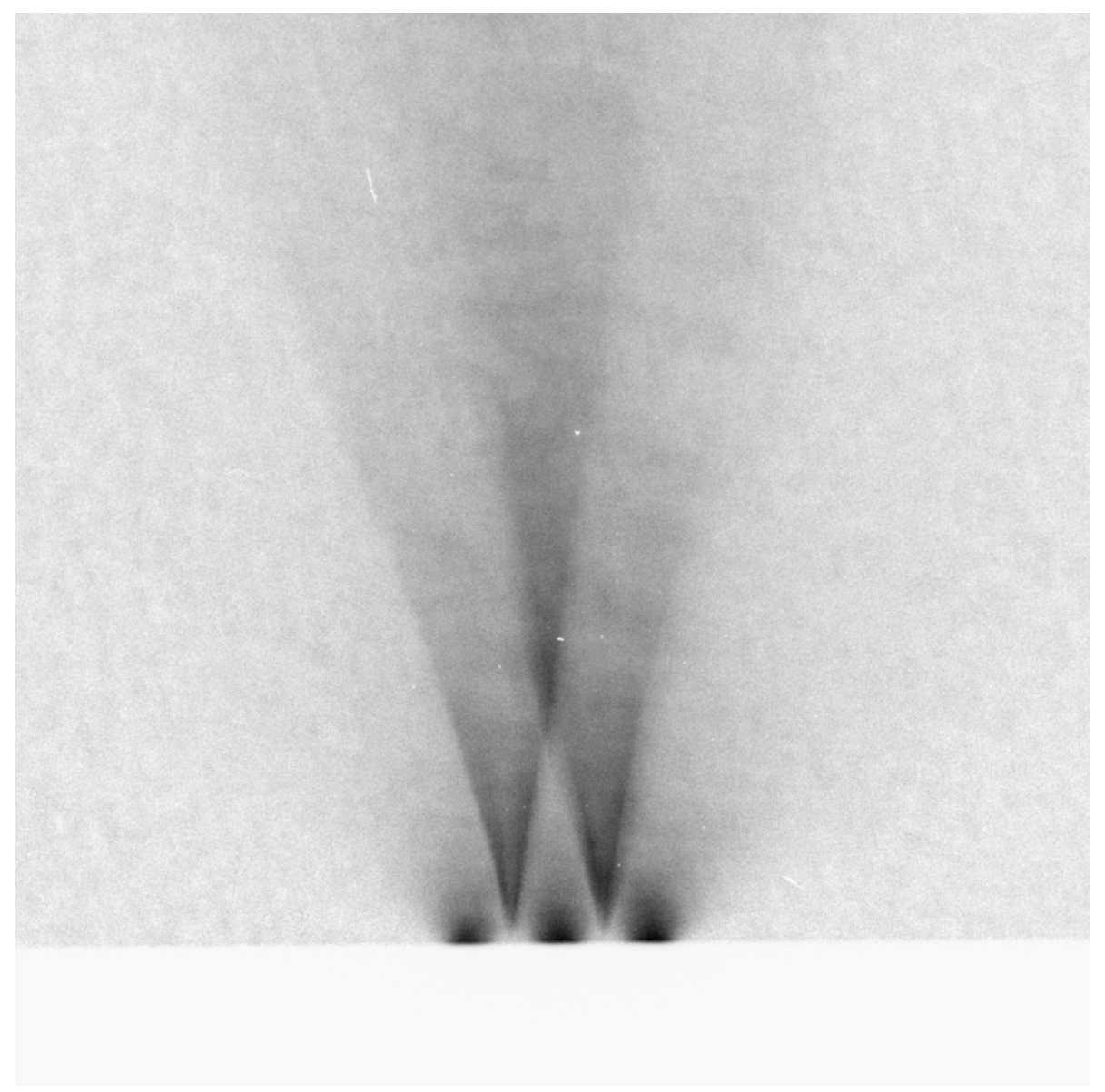

Figure 5. Raw EUV shadowgram of 3-nozzle gas jet.

obtained for a shadowgram (green curve) measured at a height of $2.6 \mathrm{~mm}$ from the nozzle exit. There is a relatively good agreement between shadowgram data and the CFD simulation result (red curve). However, a discrepancy is visible on the left shoulder of the density profile. This is due to the left nozzle releasing less gas than expected, which results in a density decrease of the left shoulder, and also causes the density spike to be less clear. The virtual gas jet produced by the two nozzles with different backing pressure is no longer normal to the nozzle surface but tilts towards the nozzle with lower pressure. This causes a shift of the secondary virtual nozzle position, leading to the discrepancy with the simulation. Another difference between experiments and simulations is due to the uncertainty in the net path length that the radiation has to travel through the gas. This distance, referred to as $d(y)$, has been estimated by assuming that the gas jet expands with an opening angle of $45^{\circ}$ and has a uniform density across the whole length. Simulations have not yet been performed for a volume with a sufficiently large lateral size to precisely estimate how $d(y)$ should behave. It is likely that $d(y)$ overestimates the shoulder density, because it neglects the lateral density drop, leading to an underestimation of the measured density. This effect is amplified when we consider the second level virtual gas jet, the density spike, which forms $2.5 \mathrm{~mm}$ above the nozzle exit and cannot have the same 
density across the whole path-length considered by $d(y)$. Finally some widening of the density spike could be attributed to EUV radiation not originating from a point source. This error can be reduced by either performing tomographic characterisation of the nozzles or by conducting simulations aimed at better understanding how $d(y)$ should behave.

\section{PIC SIMULATIONS}

Having confirmed that the gas jet density profile approximately matches the CFD simulation predictions, we study how the density profile leads to injection of attosecond electron bunches. This is undertaken using the quasi-3D particle-in-cell (PIC) code FBPIC. ${ }^{14}$ Simulations are performed for different laser spot sizes and laser energies, but with a fixed pulse duration of $25 \mathrm{fs}$, a wavelength of $800 \mathrm{~nm}$ and linear polarisation. Two simulations are presented here. Simulation 1 corresponds to a laser spot size of $20 \mu \mathrm{m}$ and normalised vector potential $a_{0}=1.8$, while simulation 2 corresponds to a laser spot size of $10 \mu \mathrm{m}$ and normalised vector potential $a_{0}=2.5$. Both simulations have $24 \mathrm{~nm}$ longitudinal resolution along the laser propagation axis $z$.

Snapshots for simulation 2 are taken at the plasma exit. Figure 6 shows the cross-section of the on-axis electron density profile. The leading electron bunch has a duration of 250 as (FWHM) followed by several bunches with a slightly longer duration, but still sub-fs. This triple bunch structure is formed at injection. The total charge in the bunch train is $37 \mathrm{pC}$. Figure 7 shows the spatial structure of the bunch, colour coded by energy, which reveals a clear negative energy chip. Finally, Figure 8 presents the energy spectrum showing three energy peaks, which are not directly correlated with the electron bunches in the train. Electrons above $150 \mathrm{MeV}$ can mainly found in the first electron bunch, whereas the peak at $100 \mathrm{MeV}$ is split between the second and third electron bunch. Simulation 1 resulted in a 250 as bunch (FWHM), but in this case a trailing high-charge electron bunch is generated at the end of the gas density plateau.

\section{CONCLUSIONS}

Clear progress towards achieving attosecond electron bunches using a laser-wakefield accelerator is presented in this paper. A triple nozzle design is presented based on CFD simulations and characterised using EUV shadowgraphy, which are in good agreement with simulations. PIC simulations based on the CDF simulation data yield electron bunches of about 250 as (FWHM).

\section{ACKNOWLEDGEMENTS}

The authors acknowledge funding from ST/R505110/1, U.K. EPSRC (EP/J018171/1, EP/N028694/1), EU H2020 Research and Innovation Programme LASERLAB EUROPE

(654148) and EuPRAXIA (653782). Credit goes to Institute of Optoelectronics, Military University of Technology in Warsaw for performing characterisation studies. Data associated with research published in this paper is available at https://dx.doi.org/10.15129/d19d2189-b759-4cd18f43-15d0369f1c84. 


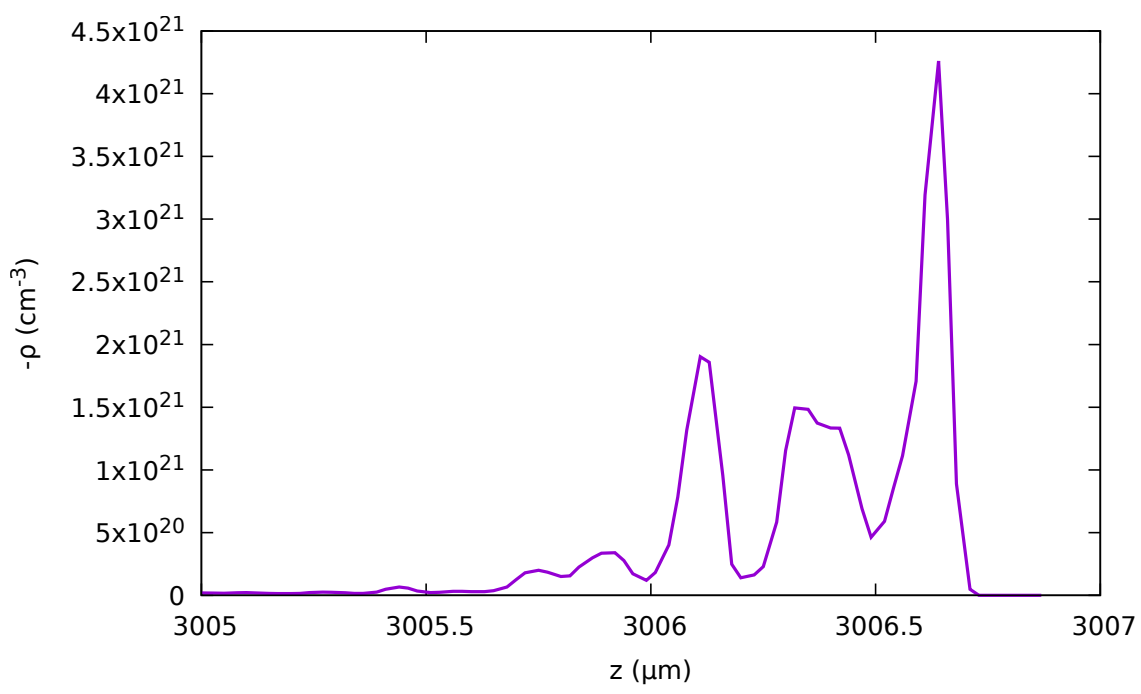

Figure 6. Longitudinal electron bunch profile generated by PIC simulations.

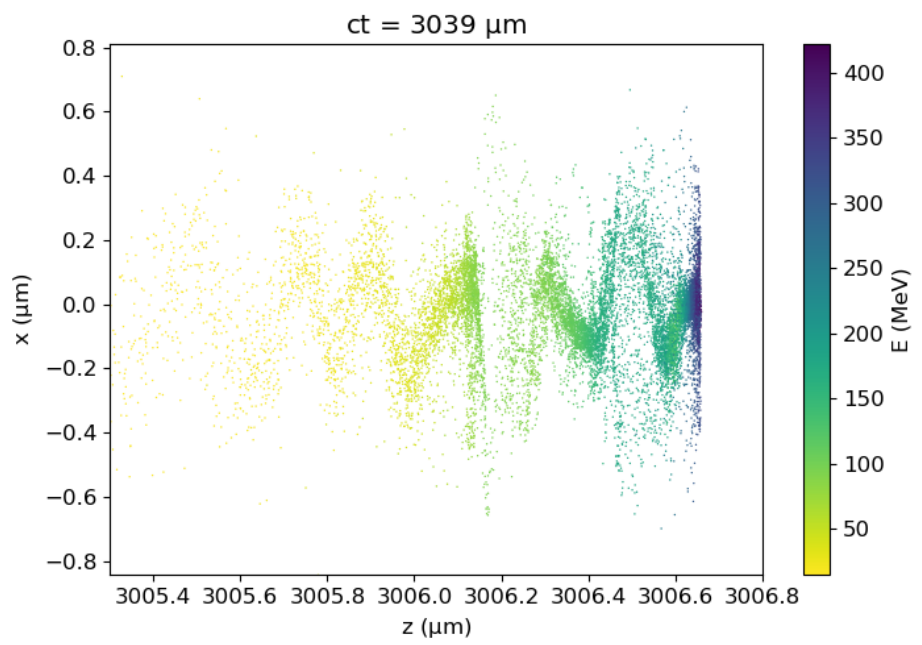

Figure 7. Spatial electron bunch structure, coloured by electron energy, generated by PIC simulations. 


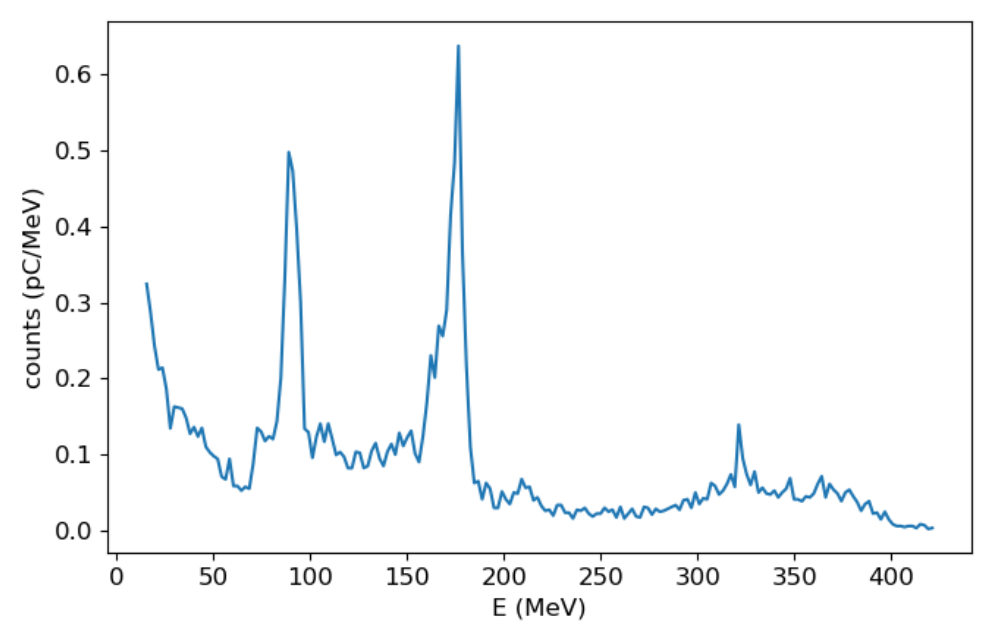

Figure 8. Electron energy spectrum generated by PIC simulations.

\section{REFERENCES}

[1] Tajima, T. and Dawson, J. M., "Laser Electron Accelerator," Physical Review Letters 43, 267-270 (July 1979).

[2] Esarey, E., Schroeder, C. B., and Leemans, W. P., "Physics of laser-driven plasma-based electron accelerators," Reviews of Modern Physics 81, 1229-1285 (Aug. 2009).

[3] Islam, M. R., Brunetti, E., Shanks, R. P., Ersfeld, B., Issac, R. C., Cipiccia, S., Anania, M. P., Welsh, G. H., Wiggins, S. M., A Noble, Cairns, R. A., Raj, G., and Jaroszynski, D. A., "Nearthreshold electron injection in the laser-plasma wakefield accelerator leading to femtosecond bunches," New Journal of Physics 17(9), 093033 (2015).

[4] Mangles, S. P. D., Murphy, C. D., Najmudin, Z., Thomas, A. G. R., Collier, J. L., Dangor, A. E., Divall, E. J., Foster, P. S., Gallacher, J. G., Hooker, C. J., Jaroszynski, D. A., Langley, A. J., Mori, W. B., Norreys, P. A., Tsung, F. S., Viskup, R., Walton, B. R., and Krushelnick, K., "Monoenergetic beams of relativistic electrons from intense laser-plasma interactions," Nature 431, 535-538 (Sept. 2004).

[5] Jaroszynski D.A, Bingham R, Brunetti E, Ersfeld B, Gallacher J, van der Geer B, Issac R, Jamison S.P, Jones D, de Loos M, Lyachev A, Pavlov V, Reitsma A, Saveliev Y, Vieux G, and Wiggins S.M, "Radiation sources based on laser-plasma interactions," Philosophical Transactions of the Royal Society A: Mathematical, Physical and Engineering Sciences 364, 689-710 (Mar. 2006).

[6] Jaroszynski, D. A. and Vieux, G., "Coherent Radiation Sources Based on Laser Plasma Accelerators," AIP Conference Proceedings 647, 902-914 (Nov. 2002).

[7] Dawson, J. M., "Nonlinear Electron Oscillations in a Cold Plasma," Physical Review 113, 383-387 (Jan. 1959).

[8] Tooley, M. P., Ersfeld, B., Yoffe, S. R., Noble, A., Brunetti, E., Sheng, Z. M., Islam, M. R., and Jaroszynski, D. A., "Towards attosecond high-energy electron bunches: Controlling self-injection in laser-wakefield accelerators through plasma-density modulation," Phys. Rev. Lett. 119, 044801 (Jul 2017). 
[9] Bulanov, S., Naumova, N., Pegoraro, F., and Sakai, J., "Particle injection into the wave acceleration phase due to nonlinear wake wave breaking," Physical Review E 58, R5257-R5260 (Nov. 1998).

[10] Menter, F., "Menter, F.: Two-Equation Eddy-Viscosity Transport Turbulence Model for Engineering Applications. AIAA Journal 32(8), 1598-1605," AIAA Journal 32 (Sept. 1994).

[11] Wachulak, P. W., Bartnik, A., Jarocki, R., and Fiedorowicz, H., "Characterization of multi-jet gas puff targets for high-order harmonic generation using EUV shadowgraphy," Nuclear Instruments and Methods in Physics Research Section B: Beam Interactions with Materials and Atoms 285, 102-106 (Aug. 2012).

[12] Fiedorowicz, H., Bartnik, A., Jarocki, R., Kostecki, J., Krzywiński, J., Mikołajczyk, J., Rakowski, R., Szczurek, A., and Szczurek, M., "Compact laser plasma EUV source based on a gas puff target for metrology applications," Journal of Alloys and Compounds 401, 99-103 (Sept. 2005).

[13] Wachulak, P. W., Węgrzyński, Ł., Zápražný, Z., Bartnik, A., Fok, T., Jarocki, R., Kostecki, J., Szczurek, M., Korytár, D., and Fiedorowicz, H., "Extreme ultraviolet tomography of multi-jet gas puff target for high-order harmonic generation," Applied Physics B 117, 253-263 (Oct. 2014).

[14] Lehe, R., Kirchen, M., Andriyash, I. A., Godfrey, B. B., and Vay, J.-L., "A spectral, quasi-cylindrical and dispersion-free Particle-In-Cell algorithm," Computer Physics Communications 203, 66-82 (June 2016). 